\title{
SHIELDING MEASUREMENTS OF THE SPACE SHUTTLE (ENDEAVOUR IN A CHANGING EMI SPACE)
}

\author{
Diane R. Kempf and Kenneth (Buzz) Brezinski \\ Naval Air Warfare Center, Patuxent River, Maryland
}

\begin{abstract}
Testing was conducted on the NASA Space Shuttle Orbiter, Endeavour, to determine the high frequency shielding capability of three major cavities; the flight deck, the mid-deck/crew quarters and the payload bay. Testing was performed using highly modified reverberation techniques.
\end{abstract}

A big advantage of reverberation is that it avoids the inaccuracies that occur during testing using traditional direct radiation methods. These include inaccuracies associated with standing wave patterns, radiation aspect angle, and antenna factor errors. Reverberation is especially useful in measuring leakages from points that are difficult to access, such as leakages from the top of the Orbiter. Reverberant technology has been demonstrated to be statistically highly repeatable and uniform over various reverberant environments. The methodology was first evaluated in an "ideal" reverberation chamber and found to be a viable solution. Parameters were refined and then it was evaluated in a "worst case" chamber at the Naval Air Warfare Center (NAWC) Patuxent River Shielded Hangar prior to testing of the spacecraft. The hangar at the Shuttle Landing Facility at Kennedy Space Center served as the "reverberation chamber" for the spacecraft.

An account of the test method is presented in detail, along with setup diagrams, photos, and graphs of the shielding measurements. Also, the software used to automate the measurements will be described. This was essential to maximizing the accuracy and efficiency of the measurements.

\section{Background}

Most military and commercial EMI limits were developed with sound engineering and a measure of conservative guessing which sometimes results in over design and added weight. This translates to higher costs for design, materials, and production. Present market trends are pushing for design to the platform and knowing the environment where you will "plug in". To accomplish such objectives, it is imperative to characterize the shielding effectiveness of the platform. The only comprehensive shielding evaluation conducted on the National Aeronautics and Space Administration (NASA) Space Shuttle Orbiter was in the early 1980 's, but has been highly scrutinized. As a result of the Columbia accident, NASA made plans to implement much higher power tracking radars to identify potential debris and subsequent tile system damage during night launch. NASA leadership recognized that new approaches to EMI are imperative and have demonstrated their commitment to change with a recent program to examine the shielding of the Space Shuttle Orbiter. This paper discusses the 4.2 to $18 \mathrm{GHz}$ frequency range that NAWC Pax River was responsible for in a joint venture with NASA and the National Institute of Standards and Technology (NIST). NIST was primarily responsible for the lower frequencies, but also provided consultation for the higher frequency range. The methodology is a progression of ideas developed by NAWC, the Naval Surface Warfare Center (NSWC), NIST and NASA. [1-4]. The changing avionics marketplace is relying on increased partnerships such as this to meld industry expertise and realize elevated goals. This "endeavour" is a case study of a truly unique EMI after-the-fact avionics-related evaluation in a new world of doing business.

\section{The Equipment Under Test}

The Space Shuttle Orbiter, specifically the Endeavour, is one of NASA's reusable space vehicles and is approximately 122 feet long, 57 feet high (on runway) and has a wingspan of 78 feet. The Orbiter consists of several cavities not limited to; the flight deck/aft flight deck, mid deck/crew compartment, payload bay, and avionics bays. The flight deck contains six pressure windshields, two overhead windows, and two rear-viewing payload bay windows. The crew compartment is a

\section{U.S. Government work not protected by U.S. Copyright}


pressurized working, living, and stowage compartment consisting of 2525 cubic feet in the forward portion of the Orbiter. The payload bay is approximately 60 feet long and 15 feet in diameter. The doors to the payload bay are on the top portion of the fuselage and are 60 feet long. The Orbiter was not in a true flight configuration, but all altered/missing components were examined and it was determined that the test configuration would yield a worst case.

\section{Test Methodology}

Testing was performed using a state of the art version of reverberation methods. Traditionally, a reverberant shielding effectiveness test is performed using "nested reverberation chambers." The field in each chamber is "stirred" using an electrically large metallic tuner that is capable of suitable displacement at the lower frequency range of the test. Statistically, this "stirring" produces a very uniform field over a revolution of the tuner. Since it is physically impractical to construct and locate a tuner that would be large enough to be effective to use in a chamber of the size needed, this test uses several antennas placed around the aircraft to provide spatial sampling instead of displacement sampling [3]. Another array of antennas is placed inside the aircraft that are moved to various locations for internal spatial sampling. Frequency averaging, or electronic stirring, is used to simulate the physical boundary movement of the RF standing wave patterns and augment the statistical sampling in the cavity [2]. As in the traditional methods, the received levels are compared to those received when the aircraft is not present (the reference measurements). The difference is the shielding effectiveness of the aircraft. A big advantage of this method is that it avoids the inaccuracies associated with conventional direct radiation methods caused by: standing wave patterns, radiation aspect angle, and antenna factor errors. These usually render very inaccurate and unrepeatable measurements. Reverberant technology has been demonstrated to be statistically highly repeatable and uniform over various reverberant environments.

\section{Software}

All data was gathered with the NAWC EMI Laboratory's Aircraft Shielding Effectiveness Software, written with HP VEE software. Simplistically, the software controls a matrix of transmit and receive signals of any size to the present limitations of 6 X 6 (six transmit antennas and 6 receive antennas). The number of receives is a result of the number of spectrum analyzers available. The transmit antennas are controlled using an IEEE-488 bus controlled RF high power switcher. Each transmit and receive can be uniquely identified, either with a name or a number. The software allows input for all associated calibration and test factor files; transmit cables, receive cables, pre-amplifiers, switches, and an auxiliary transmit factor, and noise floor information. Either linear or log stepped scans are available. Each frequency step defines a processing frequency window or "frequency stir span". The stir span is a frequency band that is approximately a half step to the left of the center frequency step to a half step to the right of the frequency step. Within this band are the "frequency stir steps". In this case, the software scanned the frequency range from $4.2 \mathrm{GHz}$ to $18 \mathrm{GHz}$ at $100 \mathrm{MHz}$ steps. The stir step size was $5 \mathrm{MHz}$ within the $100 \mathrm{MHz}$ window. Data gathered across this processing window was averaged. Typically, peak levels are gathered during reverberation, but NAWC opted for averaging. Averaging tends to be much more statistically repeatable with fewer test samples and converges much more quickly than peak data. Upon entering each frequency step stir cycle, power was leveled to a predetermined user input value.

All data was normalized to this input power (essentially an insertion loss measurement). This also allowed for flexibility of adjusting power anytime during the test process to optimize dynamic range and assure preamplifiers/amplifiers were not driven to compression. This feature also allowed data processing of the files of different power sets without need for additional compensation. In additional to all the raw corrected data, there were two types of processed data; a peak data file and an average data file. This allowed real time monitoring of "apparent" shielding data updated after each complete frequency stir measurement cycle consisting of frequency stirring with all transmit antennas. All data was normalized to a 
"default reference measurement" for this real time viewing. Several laboratory software packages were used in post data processing; a multiple file merge, multiple file averaging, multiple file peak processing, and a multiple file Chi square arrangement algorithm. This assisted in merging multiple files to the final processed values and to examine data distributions to assure statistical conformity and uniformity. The multi-file averaging algorithm was the primary software for final Orbiter data processing. This post processing performs the linear average on all the files identified.

\section{Test Validation-An Evolution}

Existing NAWC software was heavily modified to incorporate the particulars for the Orbiter and the non-ideal test environment, as well as to improve accuracy and efficiency to meet limitations on the test time and the field levels associated with the Orbiter. In a relatively ideal chamber, trial runs were performed using a $3 \times 3$ test matrix ( 3 transmit and 3 receive antennas) to examine all aspects of the software and to assure it was operating correctly. Various frequency windows, step rates, stir step rates were examined to determine suitability for the project. A $100 \mathrm{MHz}$ step fixed rate and a $5 \mathrm{MHz}$ fixed stir step were chosen for overall efficiency, accuracy, and ability to identify resonant structures with the largest possible averaging window. Averaging proved to converge many times faster than the peak values (See Figure 1) as shown for a typical shielding test and even with a limited data set (120 independent samples). A typical peak test requires thousands of samples to achieve a reliable expected value.

However, averaging is at the expense of about 20 $\mathrm{dB}$ of dynamic range. Thus, high gain, low noise pre-amplifiers were incorporated to offset the dynamic range loss of averaging. Individual matrix elements (individual X, Y: transmit, receive files) tracked very closely with only the frequency averaging. This is demonstrated in Figure 2. The standard deviation of individual matrix elements was predominantly within a $2-2.5 \mathrm{~dB}$ window.

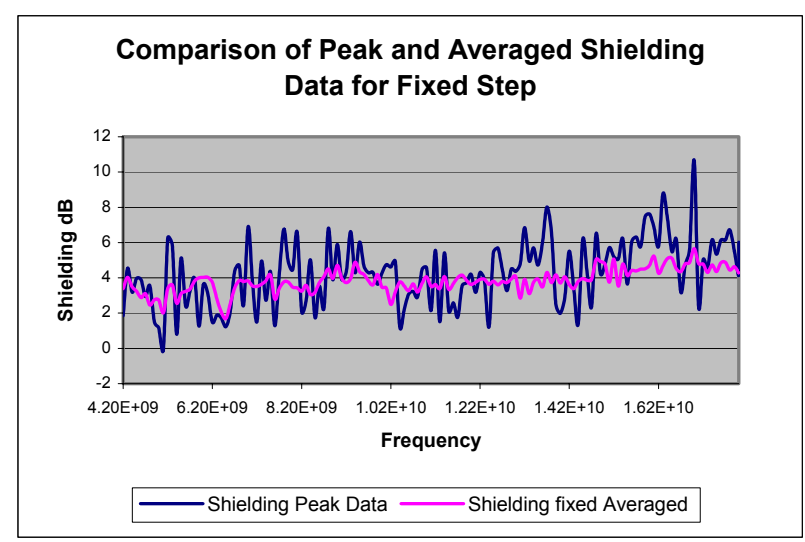

Figure 1. One Test File Comparison of Peak and Average

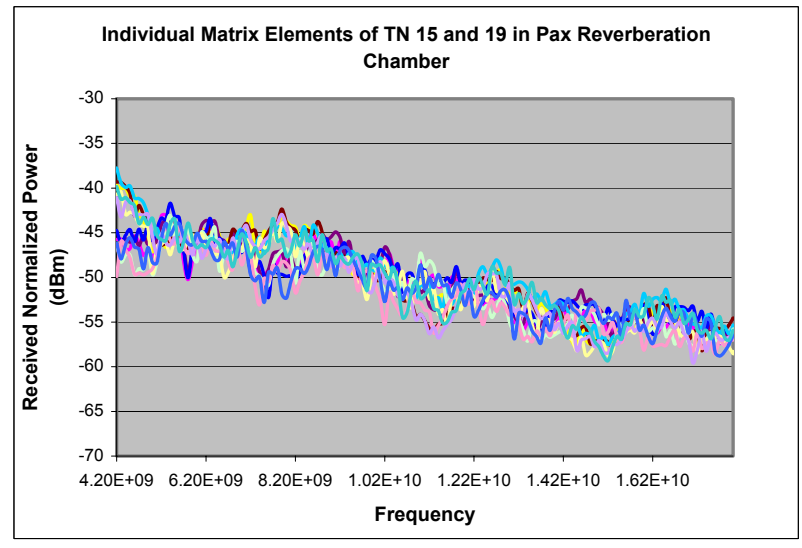

Figure 2. Data Elements of Two Different Tests

Typically, the larger the averaging frequency window, the smoother the results. Testing conducted with frequency windows approaching $\lambda / 4$ demonstrated even tighter response, however, such large windowing averages wash out cavity resonant effects. Averaging the individual matrix elements together further enhances stability as demonstrated by Figure 3 where three different tests are compared. The receive antennas from one test to another were moved and run on different days. Here the individual matrix elements from a test are averaged and compared to other averaged received power data from two other tests. The three independent test sets demonstrate very good agreement. 


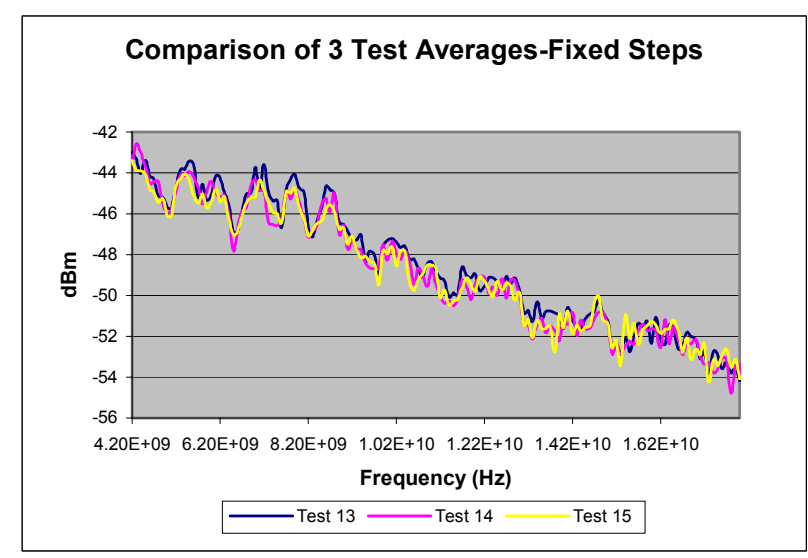

Figure 3. Averaged Data Tracking Over 3 Independent Tests

The statistical data distribution is a good indicator of the integrity of a reverberation chamber. For received power in a reverberation chamber, this is characterized with the $\mathrm{Chi}^{2}$ cumulative probability distribution with 2 degrees of freedom. Plots of an $18 \mathrm{GHz}$ distribution over two sets of test data are demonstrated in Figure 4. These are plotted against the ideal $\mathrm{Chi}^{2}$ cumulative probability distribution. The distribution tracks the ideal very well, even though three different makeshift antennas (with somewhat different responses) were used to obtain the data. Throughout this study, the lower end distributions $(4.2 \mathrm{GHz})$ were also plotted. However, these typically traced the ideal well, so they are not included. The 18 $\mathrm{GHz}$ distributions tended to reveal more information about the integrity of the chamber and of the data gathered.

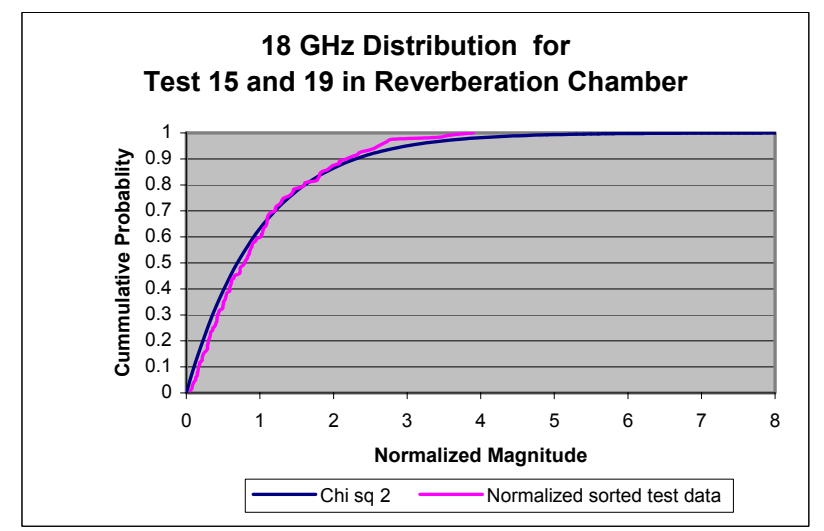

Figure 4. $18 \mathrm{GHz}$ Cumulative Probability of the NAWC Reverberation Chamber

\section{An EMI Space of a Different Kind-World's Largest Reverberation Chamber?}

A reverberation chamber of a size to support the Endeavour is simply not available. However in the past, NAWC had experimented and performed reverberation evaluations in their Aircraft Shielded Hangar with good results $[1,4]$. An evaluation in this hangar would provide the opportunity to evaluate a worst-case scenario, as the hangar is heavily loaded with a number of wooden structures, aircraft and large panels of anechoic material. Luckily, a Navy aircraft project provided an opportunity for this "small scale Endeavour", an excellent adaptation of resources to accomplish test objectives in a changing business place. Prior to the aircraft arrival, various reference tests were conducted in numerous locations about the hangar. Each test consisted of a $3 \times 4$ matrix of transmit and receive antennas. Immediately apparent, the data distributions of the individual $\mathrm{x}, \mathrm{y}$ matrix elements were much more widely spaced than was experienced in the "more ideal" reverberation chamber. This was likely due to the greater wave travel times and more prominent mass absorption due to multiple bounces when antennas were aimed towards the far walls (curved sides) of the hangar where buildings and absorber material were located. The stronger signals were merely more direct received signals. These signals encountered less space loss and less absorption by hangar contents. Figure 5 demonstrates the individual elements of two tests in the hangar showing the range of data, which had a standard deviation of typically within $5 \mathrm{~dB}$.

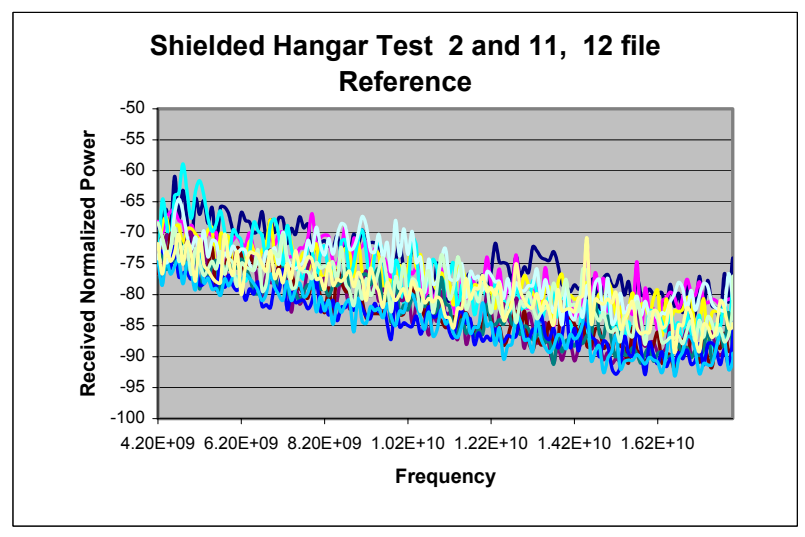

Figure 5. Data Elements of Two Different Tests in Shielded Hangar 
Figures 6 demonstrates the statistical Chi2 distribution of these tests for $18 \mathrm{GHz}$. As expected, there was a bit of departure from ideal where the variations in path lengths begin to distort the 18 $\mathrm{GHz}$ waveform.

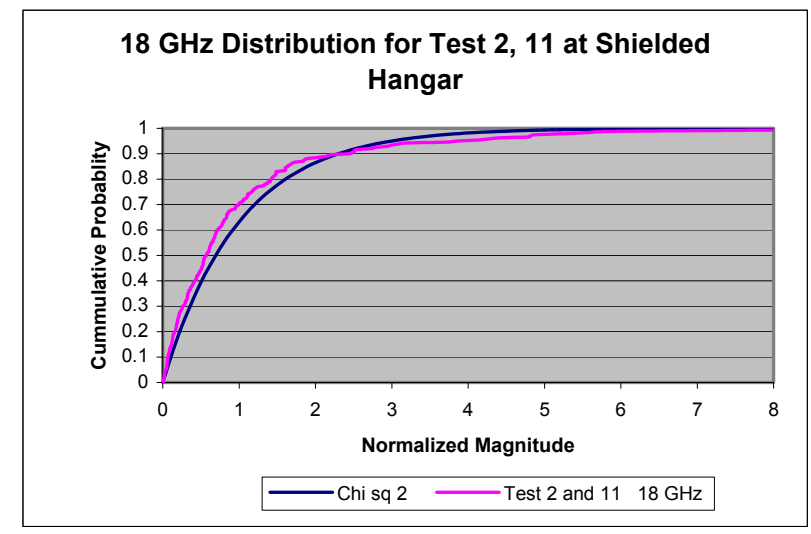

Figure 6. 18 GHz Cumulative Probability Distribution with Wire Antennas

Up to this point, evaluations in the Pax River Shielded Hangar were conducted with monopole transmit antennas fabricated by stripping the shield and connector from one end of a high frequency RF cable, an adaptation of available resources. The use of double ridge-guided horns was anticipated with the Orbiter testing, so an evaluation was conducted with these. Figure 7 demonstrates the individual elements of two tests of data taken using horns in the hangar showing the range of frequencyaveraged data.

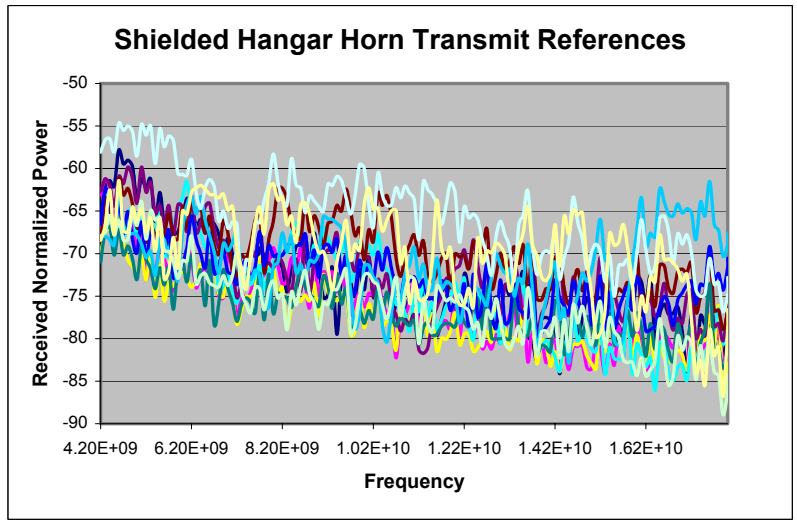

Figure 7. Data Elements of Two Different Tests in Shielded Hangar with Horns

The standard deviation of these data was typically within $5 \mathrm{~dB}$, but some values well exceeded that value, a considerable departure from an ideal chamber. Clearly, it was not as uniform as the wire antennas. Figure 8 demonstrates the statistical distributions of these tests for $18 \mathrm{GHz}$. The horn antenna response demonstrates a wider deviation of the data and distortion of the $18 \mathrm{GHz}$ distribution than the wire antenna. This is possibly due to excessive losses in areas of the hangar due to wooden buildings, absorber material, etc. and very good reflections elsewhere. A non-directional antenna may provide some statistical smoothing. In a typical room sized reverberation chamber at frequencies below $1 \mathrm{GHz}$ [1], it has been shown that there is very little difference between a directional and non-directional antenna, but above that, the direct and the reflected signal become very different due to the greater absorption of materials in the reverberation chamber. However, these findings of the omni directional antenna would appear to contradict the requirement for a directional antenna in a typical reverberation chamber. This is most likely the result of the widely varying path lengths and the resultant displacement of the individual elements of the test matrix (each transmit/receive element), i.e. the signal must travel quite a bit further in a large chamber to establish the reverberation modal structure sufficiently. Early reflections are a bit like being in the direct beam width in a smaller chamber and are very easily encountered, but hard to predict. The omni directional antenna radiates energy in many directions, which appears to reduce/break up the effect of the varying path lengths in establishing the reverberation modal structure. However, care must still be taken to avoid direct coupling.

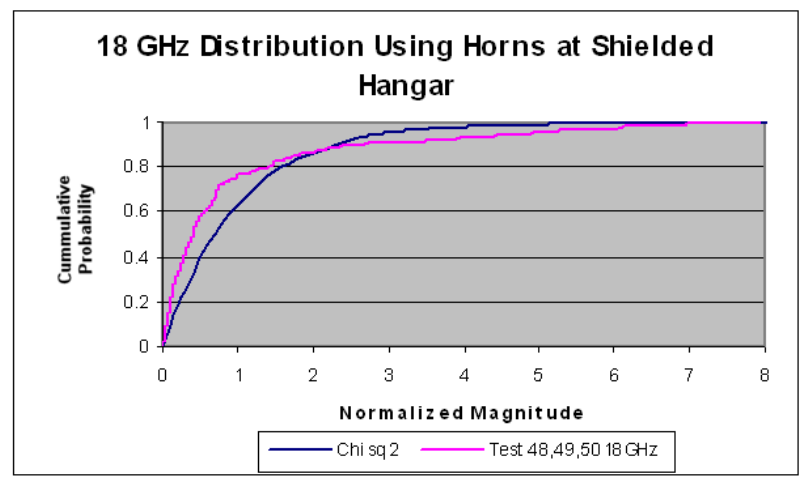

Figure 8. 18 GHz Cumulative Probability Distribution with Horn Antennas 


\section{The Holey Artifact and a Valid Space}

The poor distribution of the data using horns required additional evaluation of the "worst case" chamber. To demonstrate the final validity of test results for this methodology, NAWC was able to obtain a shielding artifact from NIST and demonstrated that this method gives the same results as those using the traditional nested chamber method in a reverberation chamber. The shielding artifact is a metal box, developed jointly by NIST and NSWC. It is an aluminum enclosure, measuring $0.73 \mathrm{~m} \mathrm{X} 0.93 \mathrm{~m} \mathrm{X} 1.03 \mathrm{~m}$, with a tuner inside. Each of the six sides contains five randomly drilled circular holes, $1.6 \mathrm{~cm}$ in diameter, to provide internal to external coupling. NAWC performed the shielding test in the large Shielded Hangar. To test the artifact, NIST used the nested chamber method in their reverberation chamber to take extensive data. Even with using only one location for the receive antennas in the hangar and three tuner positions inside the NIST box, giving a total of just 12 data sets (240 samples) for each frequency, the shielding values agreed very closely with those of NIST, verifying the method with the proven, accepted, traditional reverberation method. Figure 9 shows the shielding of the artifact box. The NIST measurements are the peak values recorded for each frequency. This shows the variability of the NIST data with frequency before averaging. Figure 9 also shows a 21 period moving average of the NIST measurements, and the NAWC measurements. Even with what would be considered a very limited data set, the curve of the NAWC data follows that of the average NIST data quite closely. So, in spite of the non-ideal standard deviation and distribution of the data in that Shielded Hangar, the results were excellent. However, great care should be taken to avoid high bias early reflections. The averaging process appears to provide consistency in spite of a nonideal distribution.

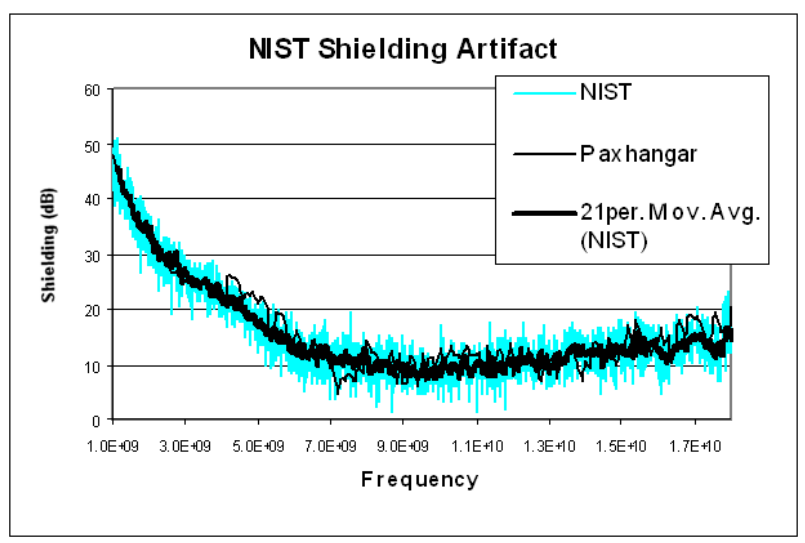

Figure 9. Shielding of the NIST Artifact

\section{Test Site}

\section{Space Port Florida-The Final Space}

There was necessarily a concern about where the "Endeavour" might take place. Originally, a huge metallic tent was proposed around the Orbiter in the Vehicle Assembly Building. However, in tradition of changing business practice, NASA skillfully negotiated the use of Spaceport Florida, called the Shuttle Landing Facility (SLF) Hangar by NASA personnel. The SLF is a state owned facility on the Kennedy Space Center complex already in use by hurricane displaced NASA personnel. This hangar measures 250 feet by 201 feet, with a curved ceiling, 65 feet high at the center. The walls and ceiling are constructed of metal and covered with foil backed fiberglass insulation and the floor is concrete. Equipment and personnel were arranged along each side of the hangar for other NASA work and they co-existed with the test personnel through test progression. Therefore, the test area was limited to approximately the middle half of the hangar. It was befitting that the hangar that had housed the Columbia wreckage investigation would provide a test platform for the shielding test to assist with Return to Flight night launch.

\section{Evaluation of the SLF "Reverberation Chamber"}

During January 18 through 21, 2005, the Pax River EMI Lab, NASA and NIST performed measurements in the SLF Hangar in order to determine its suitability for the shielding 
effectiveness test of the Orbiter. NAWC performed brief measurements with limited equipment at a few spot frequencies. NASA supplied the equipment and test personnel to operate the equipment according to the NAWC Test Plan. Of main concern was to determine its uniformity, and to obtain a rough measure of its cavity insertion loss.

Measurements were performed at 4, 8, 12, and $18 \mathrm{GHz}$ at four locations in the hangar without the Orbiter present. The field uniformity was typically $+2.5 \mathrm{~dB}$, with a worst case of $+4 \mathrm{~dB}$. Since it was simpler to perform peak measurements with the instrumentation used, the average received power was calculated using NIST published peak to average ratios for a reverberation chamber [5]. The hangar insertion losses are as shown in Table 1. Note that these are only approximate, based on the uncertainties involved with calculating an average from peak measurements with a limited amount of data.

Table 1. SLF Insertion Loss

\begin{tabular}{|c|c|}
\hline Frequency & Insertion loss \\
\hline $4 \mathrm{GHz}$ & $66 \mathrm{~dB}$ \\
\hline $8 \mathrm{GHz}$ & $75 \mathrm{~dB}$ \\
\hline $12 \mathrm{GHz}$ & $81 \mathrm{~dB}$ \\
\hline $18 \mathrm{GHz}$ & $86 \mathrm{~dB}$ \\
\hline
\end{tabular}

\section{Orbiter Shielding Effectiveness Test}

\section{Equipment Layout}

A layout of the Orbiter Shielding Test equipment is provided in Figure 10. Two computer test systems were used. Although the systems were identical and $100 \%$ interchangeable, one was designated the control and the other the processing station. These were linked together via link network to share files. The horn antennas were oriented randomly, but logically to assure good statistical data. Optical links were used to control the two receive towers, which consisted of two spectrum analyzers, two preamplifiers, two antennas, an uninterruptible power supply, an optical link and cabling. A third spectrum analyzer was included on one tower to monitor ambients. Separate $200 \mathrm{ft}$ power cords fed each of the receive towers, the processing station, and the transmit rack. This allowed free independent movement of all components. Each tower was fed with an independent 60-foot optical control link. A separate optical link was provided to the transmit rack, containing the synthesizer, amplifier, and RF switch.

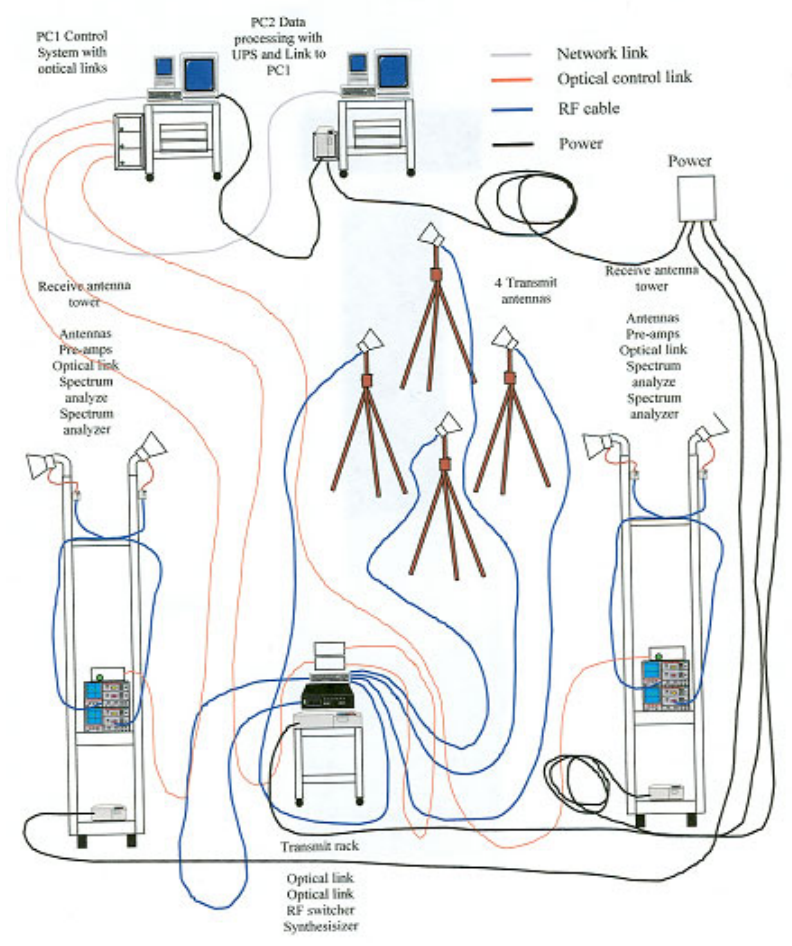

Figure 10. Shielding Equipment Layout

Of course, this transmit system was only on a cart when conducting reference measurements outside the Orbiter. When inside the Orbiter, it was split to facilitate better movement to the various test locations. The transmit antennas were taken off the tripods and mounted to a single antenna stand in the Orbiter. All cables in the Orbiter installation used prodigious quantities of ferrites to prevent RF feedback, including power cord penetration into the Orbiter and between cavities. Power in the Orbiter was filtered prior to feeding equipments. Figure 11 shows a typical set up of control/processing relative to receive towers. The two towers were typically close enough to the processing stations to provide for quick observations of signals relative to noise floor. 


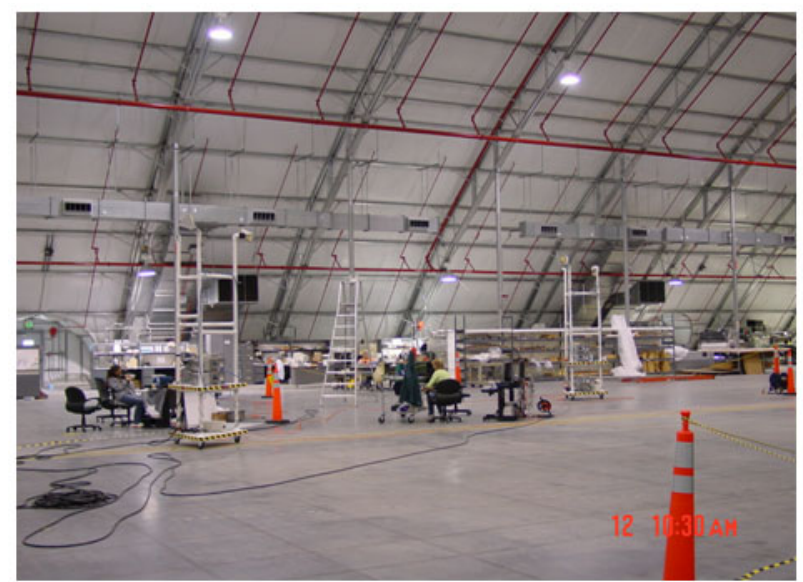

Figure 11. Typical Layout of Control and Processing System Relative to Receive Tower

\section{Reference Measurements}

Reference measurements are essentially a measurement of the insertion loss of the reverberant cavity that the test item is placed in. A measured power is transmitted into the cavity, the SLF hangar in this case, and the power received by the receive antennas is recorded for each frequency. This is the baseline that the Orbiter measurements are compared to, after both sets of measurements are normalized. The difference between the reference measurements and the measurements performed with the transmit antennas inside the Orbiter is then the shielding effectiveness of the Orbiter.

Reference measurements were performed with the Orbiter in the reverberation structure. The reference runs consisted of 8 data sets; two transmit locations, with four receive locations for each, covering the 4 quadrants of the Orbiter. This resulted in 16 files ( 4 transmit $\mathrm{X} 4$ receive) at each of 8 locations with a 20 sample frequency stir step. This equals 2560 samples/frequency step. The receive antennas were typically pointed away from the Orbiter to allow for maximum reverberation of the transmit signal before it arrived at the receive antennas. This configuration avoided direct and first reflection coupling of the transmit and receive antennas, and the possible excessive absorption due to the spacecraft. Also, care was taken to avoid any symmetry in the receive antenna locations. The transmit antennas are shown in Figure 12. The receive antenna locations are shown in Figure 13.

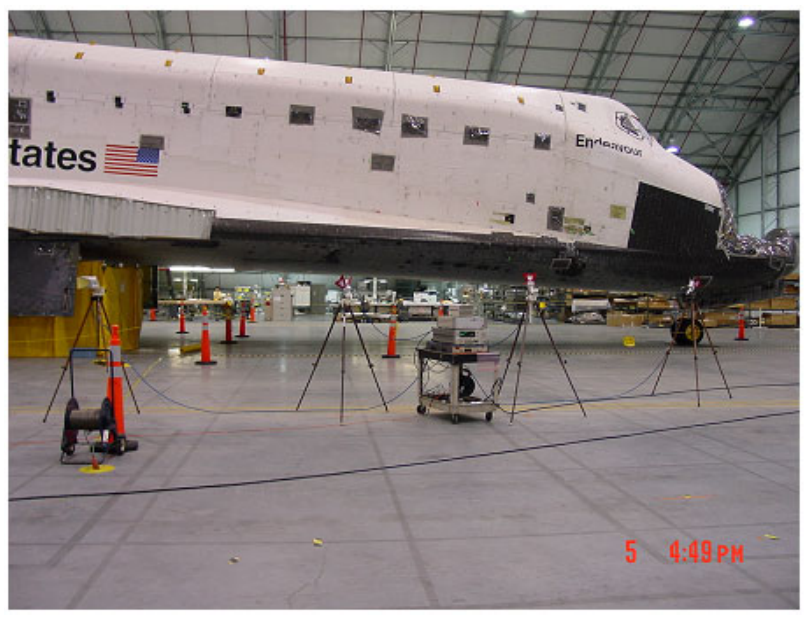

Figure 12. Reference Measurements - Transmit Antennas

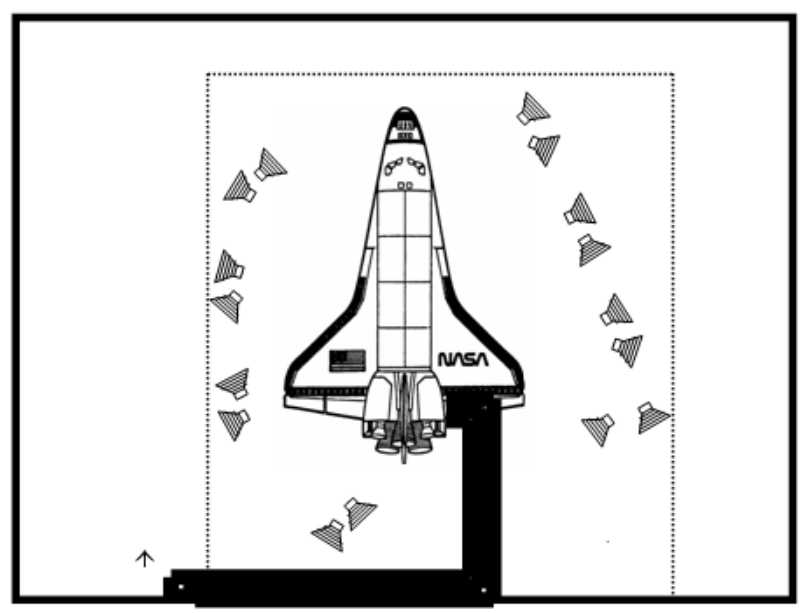

Figure 13. Receive Antenna Locations

\section{Space" \\ SLF Hangar Insertion Loss or "Loss in}

An average of all of the normalized data gathered during the reference measurements is essentially the average insertion loss of the SLF Hangar. This was used as the reference to which all of the Orbiter measurements were compared to, in order to calculate the shielding effectiveness. This is shown in Figure 14. The bounds for the 8 individual test positions that make up this data are provided in Figure 15. This is basically the uniformity of the chamber. As with other enclosure evaluations using this methodology, the cumulative probability of the reference measurements was compared to an ideal Chi2 distribution. This is provided in Figure 16. Note that there is some 
departure from the ideal, but much better than the NAWC Shielded Hangar.

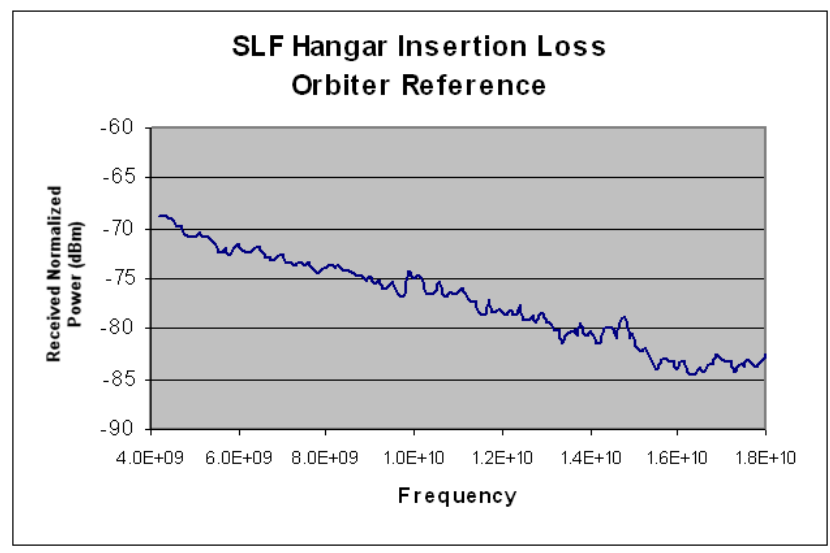

Figure 14. SLF Hangar Insertion Loss

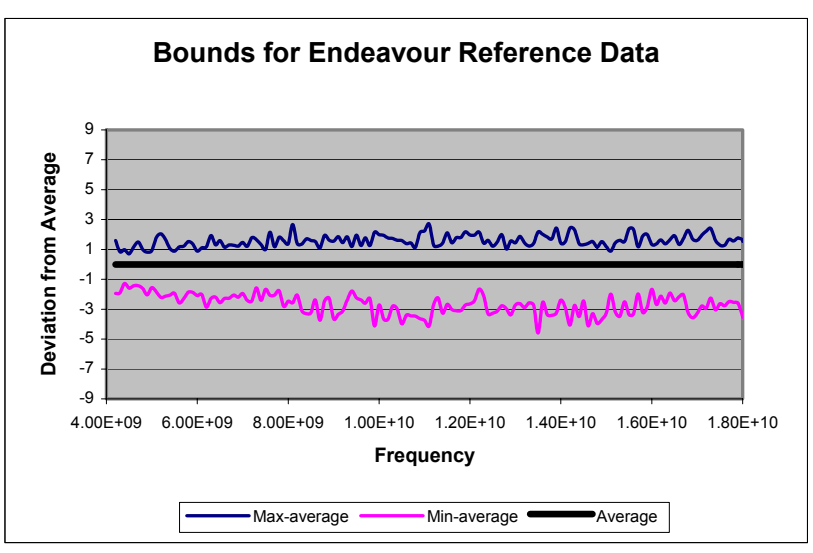

Figure 15. Bounds for Reference Data

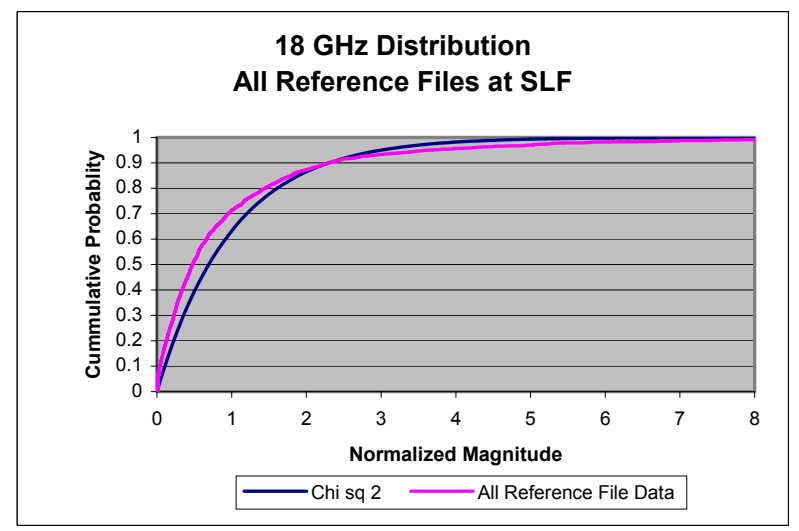

Figure 16. 18 GHz Cumulative Probability Distribution in the SLF Hangar

\section{The Test "Endeavour"}

\section{Test Set-Up}

For the Orbiter shielding test, equipment was distributed in two areas as shown in Figure 17.

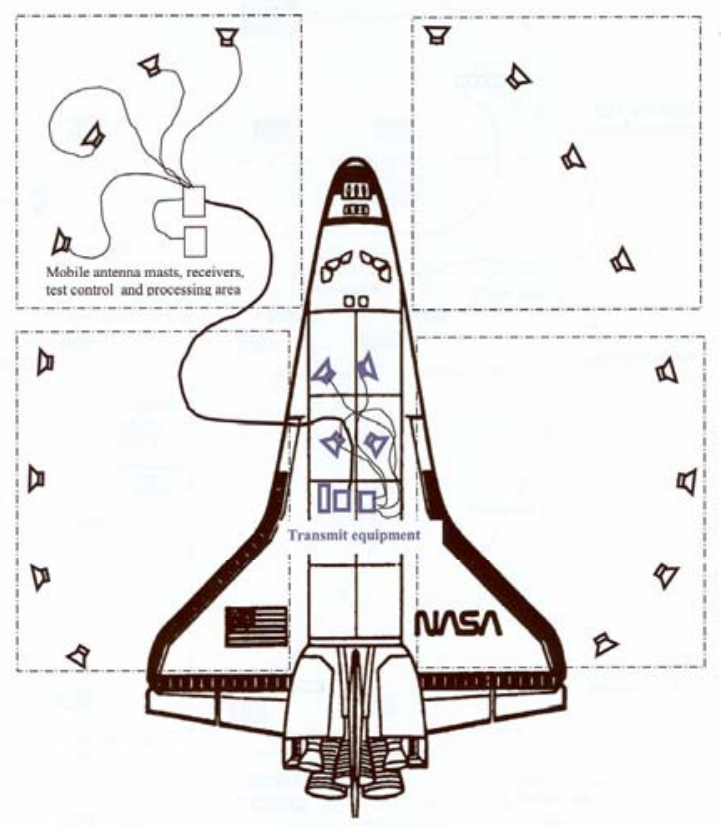

Figure 17. Equipment Setup Showing 4 Receive Test Quadrants (One at a Time)

Outside of the Orbiter were two moveable receive towers. A fiber optic link provided communication with the test computers. Four transmit antennas were located in the Orbiter cavity being tested. Figure 18 shows the approximate locations of the transmit antennas to test the three cavities. Internal antennas were asymmetrically placed about each cavity. Four antenna positions were used in each of the flight deck, and crew compartments, resulting in 16 files at each of 4 receive locations, using 20 stir frequency samples for a total of 1280 samples at each frequency step. Six transmit antenna positions were used in the payload bay section, three at a time for a total of 1920 samples at each frequency step.

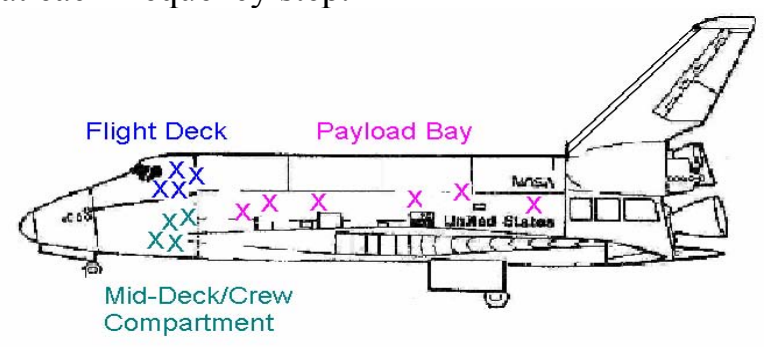

Figure 18. Transmit Antenna Locations 


\section{Results of the Orbiter Shielding Effectiveness Measurements}

The shielding results provided below demonstrate relatively flat characteristics, unlike traditional methods, which display very large swings in data from frequency to frequency. Note that this is not necessarily a result of the methodology, but this method does tend to provide smoother, more consistent results. However, resonant effects are easily identified using this methodology. These effects are normally only encountered in very tight shielding situations with highly tuned slots. Typically, broad apertures emit a range of frequencies consistently, as is the case with the Orbiter. Although the payload bay would appear to have highly tuned slots at the seams of the doors, they are most likely much longer than the wavelengths of the frequencies involved, so they tend to attenuate consistently across the 4.2 to 18 $\mathrm{GHz}$ band.

The results are presented as relative numbers, since at the time of this paper submittal; an official report of the testing had not yet been released.
NIST data overlapped that of NAWC in the frequency range of 4.2 to $6 \mathrm{GHz}$. Interestingly, even with two completely different test methodologies, there was consistently good agreement between like sets of data in insertion loss, uniformity and shielding values.

\section{Flight Deck}

The average shielding results for the flight deck are shown in Figure 19. This shows the average shielding measured with all four transmit antennas. The range of the shielding was approximately $4 \mathrm{~dB}$ throughout the 4.2 to $18 \mathrm{GHz}$ frequency range. Figure 19 also shows the shielding measured using transmit antenna \#1 only, which was aimed directly out the flight deck window. In this case, the shielding was approximately $3 \mathrm{~dB}$ lower than when all four transmit antennas were averaged together. This represents worst case shielding for the flight deck.

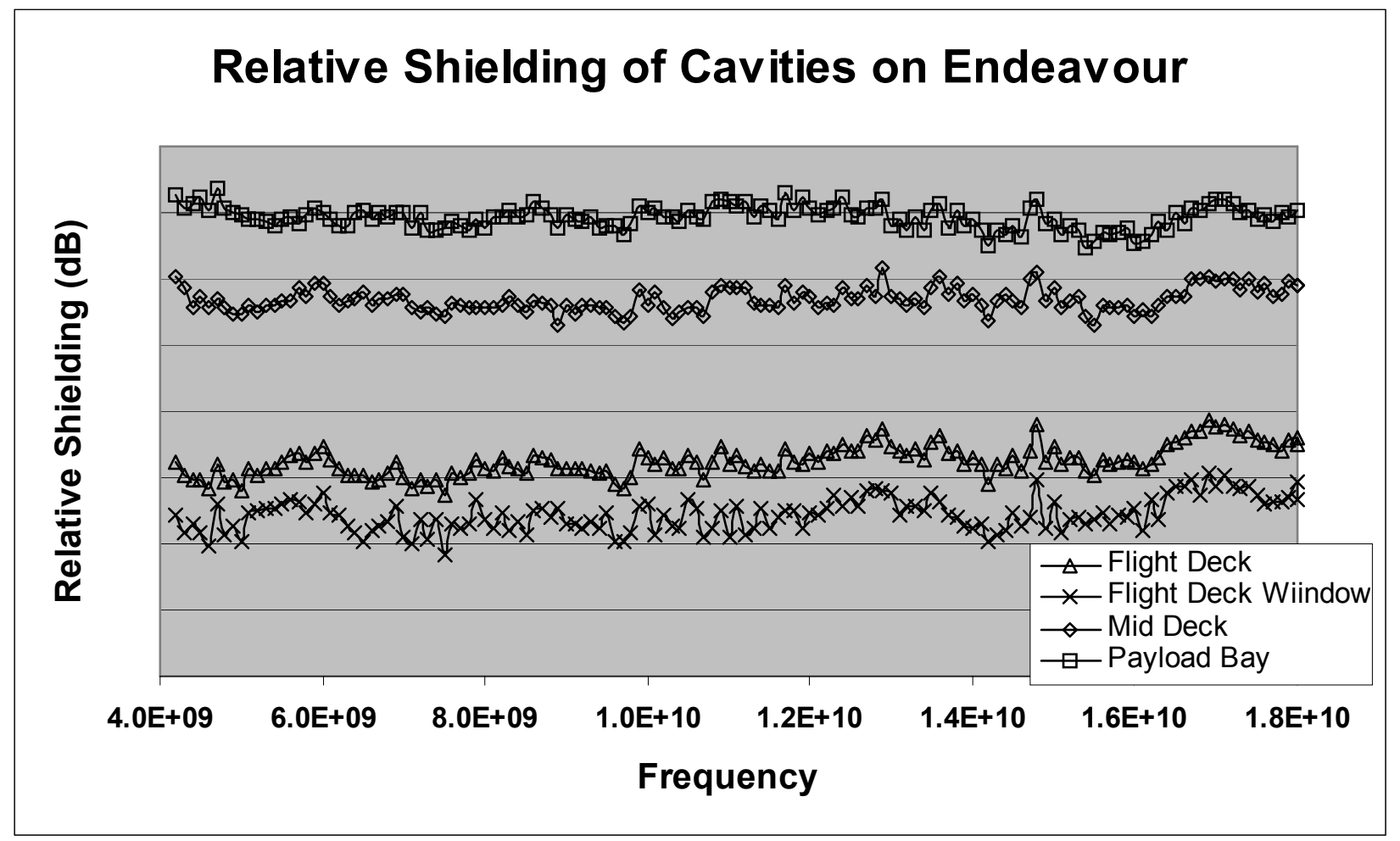

Figure 19. Relative Orbiter Shielding 


\section{Mid-Deck}

The shielding of the Orbiter in the mid-deck in the 4.2 to $18 \mathrm{GHz}$ frequency range is shown in Figure 19. The range of the shielding was approximately $4 \mathrm{~dB}$ throughout the 4.2 to $18 \mathrm{GHz}$ frequency range.

\section{Payload Bay}

While collecting data for the payload bay, the preliminary shielding values were somewhat higher than expected. Due to the large longitudinal seam along the payload bay doors that was not designed to provide RF shielding, it was expected that the shielding of the payload bay would be very poor. It was suspected that the radiation leakage pattern of the payload bay door seam might have been aimed primarily up towards the ceiling. Therefore, one receive antenna was aimed up at the peak of the ceiling in an attempt to catch the first bounce of the signals leaking out the seam of the payload bay doors. This only had an effect of about $2-3 \mathrm{~dB}$ on the shielding values. Other antenna positions were used in an attempt to determine if any particular payload bay "antenna" and hangar shape interaction was going on, but none of these additional measurements indicated anything unusual. Extensive equipment checks were performed in order to eliminate any possibility of error. It is uncertain at this time exactly why the payload bay was found to have such excellent shielding. Detailed analysis of the data have led us to some theories but without further testing, modeling, and simulations, it is premature to present them in this report. In any case, any skewing of the measurements due to unusual spacecraft/hangar interactions affecting the reference measurements and the payload bay shielding measurements differently appears to have an effect on the payload bay shielding values of only a few $\mathrm{dB}$. The shielding measurements for the payload bay are shown in Figure 19.

\section{Conclusions}

New methodologies are needed to conduct more realistic EMI evaluations of avionics equipments in this new, rapidly changing business environment. EMI spaces and practices do not necessarily need to conform to the usual paradigms.
Teamwork and innovation paid off big dividends in achieving success with this large "Endeavour".

When viable to use, average reverberation test data converges much faster and is much more tolerant of test environment than peak data gathering. Care is required to avoid dynamic range issues using averaging.

The test method of frequency stirring in a reverberant environment has been shown to be accurate compared to the nested chamber method in a reverberation chamber.

Testing in the large SLF Hangar and the Pax River Shielded Hangar has shown that there are test phenomenons unique to very large reverberation chambers that need further investigation.

\section{Acknowledgements}

The PAX River shielding test team would like to thank the entire NIST test team: Bob Johnk, David Navotny, Chris Grosvener, Nino Canales, and Dennis Camell for their insights, suggestions, and expertise. It was a pleasure working with them all. Also of NIST, we especially acknowledge Galen Koepke. With his vast experience and knowledge of reverberation methods, Galen provided valuable advice and was an excellent liaison between the NIST and Pax test teams, using two very different test methods to measure the shielding of the Orbiter. All of the folks at NASA, too numerous to name here, who coordinated the effort, supported the Orbiter, and provided assistance when needed, are all greatly appreciated. We heartily thank our Team who fully supported the "Endeavour" by getting equipment together, organizing and delivering that equipment and filling the gaps in workload back home during the "Endeavour". And last, but certainly not least, a big thanks to Bob Scully of NASA, a true leader who facilitated this Endeavour in spite of all the odds. We are grateful to be part of such a successful, interesting, project.

\section{References}

[1] Kempf, Diane R., Kenneth "Buzz" Brezinski, October 2001, Using Reverberation Techniques for Testing an Intercommunications System on a Navy 
Aircraft (2001: A Test Lunacy), 2001 IEEE Digital Avionics Conference

[2] Grosvenor, Chriss. A., Robert T. Johnk, David R. Novotny, Nino Canales, Claude M. Weil, Jason Veneman, January 2003, A Two-Phase Airframe Shielding Performance Study Using Ultra-wideband Measurements Systems, NISTIR 6622, National Institute of Standards and Technology

[3] Hatfield, Michael, April 2003, "In-Situ Shielding Measurements", Reverberation Chamber, Anechoic Chamber, OATS, and GTEM User's Meeting
[4] Kempf, Diane R., Kenneth "Buzz" Brezinski, September 2002, Shielding Effectiveness Testing of the Learjet 45, NAWC AD EMI Report

[5] Ladbury, John M.; Galen H. Koepke, June 1999, Reverberation Chamber Relationships:

Corrections and Improvements or Three Wrongs Can (Almost) Make a Right, Reverberation Chamber, Anechoic Chamber, and OATS User's Meeting

$24^{\text {th }}$ Digital Avionics Systems Conference October 30, 2005 